\title{
INFLUÊNCIA DE CHUVA SIMULADA NA EFICÁCIA DE DIFERENTES FORMULAÇÕES E DOSES DE GLYPHOSATE
}

\author{
Erivelton Scherer Roman ${ }^{1}$
}

'Pesquisador. Embrapa Trigo. Caixa Postal 451. Passo Fundo, RS 99001-970 eroman@cnpt.embrapa.br

\begin{abstract}
RESUMO
Os efeitos de chuva simulada sobre a eficácia de diferentes sais, doses e formulações de glyphosate, no controle de Brachiaria plantaginea (Link) Hitchc., foram estudados em condições de campo. O delineamento experimental foi de blocos divididos em faixas, as quais eram constituidas pelo fator irrigação. Os fatores formulações e doses foram arranjados em esquema fatorial completo, com 4 repetições. As parcelas mediam 2,5 m x 4,0 m. O fator irrigação consistiu em eventos de 20 mm de chuva simulada, aplicada em diferentes intervalos de tempo após a aplicação dos herbicidas. A simulação da chuva foi realizada com o auxílio de um sistema de irrigação por aspersão. Chuvas simuladas em intervalo de tempo inferior a 4 horas após a aplicação dos herbicidas reduziram a eficácia dos produtos, quando aplicados na dose mais baixa (360 g/ha do equivalente ácido). A atividade do sal de amônio, na formulação de grânulos autodispersíveis em água (WG), sofreu maior redução que a do sal monopotássico e a do sal isopropilamina, ambos na formulação concentrado solúvel (CS), nessa dose. O efeito negativo da chuva pode ser reduzido pelo aumento da dose do ingrediente ativo. O tipo de sal e a formulação determinaram a velocidade de controle da planta daninha. O sal isopropilamina e o sal monopotássico, ambos na formulação CS, apresentaram taxas de controle diárias estatisticamente iguais entre si e superiores às do sal de amônio, na formulação WG. Os sais e as formulações determinaram também a eficácia de controle; o sal isopropilamina e o sal monopotássico, ambos na formulação CS, apresentaram controle superior ao conferido pelo sal de amônio, na formulação WG. A eficácia de glyphosate demonstrou estar ligada ao tipo de sal, à formulação, à dose e ao intervalo de tempo entre a aplicação e a ocorrência de chuva.
\end{abstract}

Palavras-chave: Brachiaria plantaginea, herbicida.

\author{
ABSTRACT \\ Influence of simulated rain on the efficacy of different formulations \\ and doses of glyphosate
}

The effects of simulated rainfall on the performance of different formulations and doses of glyphosate in the control of Brachiaria plantaginea (Link) Hitchc. were studied under field conditions. The experimental design used was that of a split-block in strips. The strips consisted of the irrigation factor. Formulation and doses were arranged in a factorial arrangement, with four replications. Plots measured $2.5 \mathrm{~m} \mathrm{x} 4.0 \mathrm{~m}$. The irrigation factor consisted of a simulated rainfall of $20 \mathrm{~mm}$ applied at different timings (1, 2, 4 and 24 hours after treatment application) which was carried-out using a system of sprinklers. Rainfall simulated less than 4 hours after spraying the products reduced the phytotoxicity of the ammonium salt formulated as water auto-dispersible granules (WG) to a greater extent than that of the potassium and isopropylamine salts formulated as soluble concentrate (SC) when all compounds were applied at the rate of $360 \mathrm{~g} / \mathrm{ha}$ acid equivalent. Higher rates of glyphosate reduced the negative rainfall effect. The efficiency of the compound was affected by the type of salt and formulation. The isopropylamine and the potassium salts both as SC formulation, presented daily control rates higher than the isopropylamine as WG formulation. The final control of B. plantaginea was also affected by the formulation; both the 
isopropylamine salt and the potassium salt formulated as SC showed control rates higher than the ammonium salt formulated as WG. In conclusion, glyphosate efficiency is determined by its salt type, formulation, rate, and time interval between its application and rainfall. These factors affect glyphosate absorption and translocation determining the final control level of the studied species.

Key words: Brachiaria plantaginea, herbicide.

\section{INTRODUÇÃO}

A ocorrência de chuvas após a aplicação de herbicidas em pós-emergência pode reduzir a eficácia de controle de plantas daninhas por esses produtos devido à sua lavagem das folhas antes de serem absorvidos pela planta (Behrens \& Elakkad, 1981). Desse modo, o intervalo de tempo entre a aplicação do herbicida e a ocorrência de chuvas (Anderson \& Arnold, 1984), bem como a quantidade e a intensidade de chuva (Hammerton, 1967), além do tipo de formulação do produto, influem no controle de plantas daninhas conferido por esses produtos químicos.

Glyphosate é um composto polar, solúvel em água, sendo sua absorção pela planta dependente da formulação, provavelmente determinando a quantidade de glyphosate disponível para translocação, o que, dessa forma, determina seu desempenho, especialmente quando aplicado em doses abaixo do necessário para controle de determinada espécie. A maioria das formulações disponíveis de glyphosate, por exemplo, requer um período de 6 horas sem chuva, após a aplicação, para assegurar a absorção máxima pelas plantas daninhas (Rodrigues \& Almeida, 1998).

Aumentando-se a dose do produto, o intervalo de tempo entre a aplicação e a ocorrência de chuva, necessário para que o herbicida seja absorvido pela planta, pode ser reduzido (Hawf \& Behrens, 1974). É também sugerido que algumas formulações têm ação independente das condições ambientais. A formulação do herbicida e o intervalo entre a aplicação e a ocorrência de chuva foram os fatores mais importantes na redução da atividade do herbicida pela chuva, como demonstrado por Weaver et al. (1970). Produtores que usam o sistema plantio direto têm interesse em conhecer o efeito de chuvas que possam ocorrer após a aplicação de herbicidas dessecantes na eficácia dos produtos, para a tomada de decisão sobre sua aplicação.

O presente estudo teve por objetivo determinar os efeitos de chuva simulada sobre a eficácia, como dessecantes, de diferentes sais, doses e formulações de glyphosate, no controle de Brachiaria plantaginea, em condições de campo.

\section{MATERIAL E MÉTODOS}

O experimento foi conduzido em condições de campo na área experimental da Embrapa Trigo, localizada no mu- nicípio de Passo Fundo RS, no ano de 2001. O solo é de textura média, com $42 \%$ de argila e $4 \%$ de matéria orgânica, pertencente à Unidade de Mapeamento Passo Fundo (Latossolo Vermelho Distrófico típico). Os tratamentos (Tabela 1) foram aplicados em pós-emergência da planta daninha (Brachiaria plantaginea) no dia 07/02/01, usando-se um pulverizador costal de precisão, munido de bicos de jato em forma de leque, do tipo 110 02, a 1,4 kg/ $\mathrm{cm}^{2}$ de pressão de trabalho, dada por gás carbônico, e consumo de calda de 200 1/ha. A pulverização foi iniciada às 10 h10 e terminada às 11h45. No momento da pulverização o solo encontrava-se úmido, a umidade relativa do ar era de $70 \%$ e a temperatura ambiente era de $30^{\circ} \mathrm{C}$. Não ocorreu déficit hídrico para as plantas daninhas durante a condução do experimento. No momento da aplicação dos tratamentos as plantas daninhas encontravam-se com 50 a $60 \mathrm{~cm}$ de altura e com cinco a oito afilhos.

O delineamento experimental foi de blocos divididos em faixas, as quais eram constituídas pelo fator irrigação. Os fatores formulações e doses foram arranjados em esquema fatorial completo, com 4 repetições. As parcelas mediam 2,5 $\mathrm{m}$ x 4,0 m. O fator irrigação consistiu em eventos de $20 \mathrm{~mm}$ de chuva simulada, aplicada em diferentes intervalos de tempo após a aplicação dos tratamentos com herbicidas (Tabela 1). A simulação da chuva foi realizada com o auxílio de um sistema de irrigação por aspersão.

A eficácia dos tratamentos no controle (dessecação) de $B$. plantaginea foi determinada aos 3, 7, 14, 21, 28 e 42 dias após o tratamento (DAT) pelo método de avaliação visual, atribuindo-se notas em percentagem de controle em relação à testemunha. A escala empregada variou entre 0 (sem danos visíveis) e $100 \%$ (dessecação total de plantas). Os dados de eficácia dos tratamentos, depois de submetidos a testes de homogeneidade da variância e da distribuição do erro experimental (normalidade), foram transformados por meio de arco seno $[(\sqrt{\mathrm{x}+1}) / 100]$, para análise de variância. Para comparação das médias dos tratamentos usou-se o teste de Duncan, a $5 \%$ de probabilidade de erro, empregando-se o programa SAS (proc glm). Também foram realizadas análises conjuntas dos dados e teste de contrastes de interesse, para responder questões específicas sobre velocidades de ação do sal monopotássico e do sal isopropilamina, na formulação concentrado solúvel (CS), o efeito das doses de glyphosate em função do tipo de formulação do produto Comparação entre o tipo de sal e formulações e a influência do intervalo de tempo entre a aplicação dos tratamentos e a influência da 
Influência de chuva simulada na eficácia de glyphosate

Tabela 1. Tratamentos aplicados em pós-emergência no controle de Brachiaria plantaginea. Embrapa Trigo, Passo Fundo, RS. 2001.

\begin{tabular}{|c|c|c|c|c|c|}
\hline \multirow[b]{2}{*}{ Tratamento } & \multirow[b]{2}{*}{$\begin{array}{l}\text { Produto } \\
\text { comercial }\end{array}$} & \multirow[b]{2}{*}{$\begin{array}{l}\text { Formu- } \\
\text { lação* }\end{array}$} & \multicolumn{2}{|c|}{ Dose/ha } & \multirow{2}{*}{$\begin{array}{l}\text { Intervalo entre } \\
\text { a aplicação } \\
\text { e a irrigação } \\
\text { (horas) }\end{array}$} \\
\hline & & & $\begin{array}{c}\text { Equivalente } \\
\text { ácido } \\
\text { (grama) }\end{array}$ & $\begin{array}{c}\text { Produto } \\
\text { comercial } \\
\text { (litro ou kg) }\end{array}$ & \\
\hline Glyphosate (Sal monopotássico) & Zapp QI & CS & $360,720,1440$ & $0,72,1,44,2,88$ & $1,2,4$ e 24 \\
\hline Glyphosate (Sal isopropilamina) & Roundup Transorb & CS & $360,720,1440$ & $0,72,1,44,2,88$ & $1,2,4$ e 24 \\
\hline Glyphosate (Sal amônio) & Roundup WG & WG & $360,720,1440$ & $0,72,1,44,2,88$ & $1,2,4$ e 24 \\
\hline Testemunha & - & - & - & - & $1,2,4$ e 24 \\
\hline
\end{tabular}

*CS= concentrado solúvel; WG= grânulos autodispersíveis em água.

chuva simulada na eficiência da dessecação de B. plantaginea, usando-se o teste $\mathrm{F}$ e análises de regressão. A velocidade de ação dos tratamentos químicos foi expressa pela análise de regressão entre a percentagem de controle obtido e o tempo (DAT)

\section{RESULTADOS E DISCUSSÃO}

\section{Velocidade de evolução dos sintomas de fitotoxicidade do glyphosate}

A regressão entre a percentagem de controle de $B$. plantaginea obtida de dados para os produtos em estudo e o tempo (DAT) demonstrou que as velocidades de ação do sal monopotássico e do sal isopropilamina, na formulação concentrado solúvel (CS), foram estatisticamente iguais entre si e superiores à do sal de amônio, na formulação de grânulos autodispersíveis em água (WG) (Figura 1). Como a absorção de qualquer substância pela planta precede a sua translocação, a taxa de absorção determina a quantidade disponível para transporte e para o efeito final na planta. Os dados obtidos sugerem que diferenças nas taxas de absorção dos produtos (formulações) podem ter sido responsáveis pelas suas velocidades de ação, fato também verificado por Harrison \& Wax (1986) quando estudaram o efeito de diferentes adjuvantes na absorção de haloxyfop-methyl. A velocidade de ação de herbicidas dessecantes pode ser importante para o estabelecimento de culturas no sistema plantio direto, evitando a competição já nos estádios iniciais da cultura.

\section{Efeito das doses de glyphosate em função do tipo de formu- lação do produto}

A análise dos dados obtidos em todas as avaliações realizadas (Tabela 2 ) revelou que o melhor controle foi obtido com sal monopotássico na formulação concentrado solúvel
(CS), com sal isopropilamina na formulação concentrado solúvel (CS) e com sal de amônio na formulação de grânulos autodispersíveis em água (WG), na dose de 1440 g/ha e.a., os quais não diferiram entre si. Os controles obtidos com o sal isopropilamina, na formulação CS, nas doses de $720 \mathrm{~g} / \mathrm{ha}$ e.a. e de $1440 \mathrm{~g} / \mathrm{ha}$ e.a., foram equivalentes estatísticamente. Os tratamentos com a dose mais baixa (360 g/ha e.a.) de todos os sais e formulações propiciaram controle inferior ao dos demais tratamentos, independentemente do tempo decorrido entre a aplicação dos tratamentos e a ocorrência da chuva simulada.

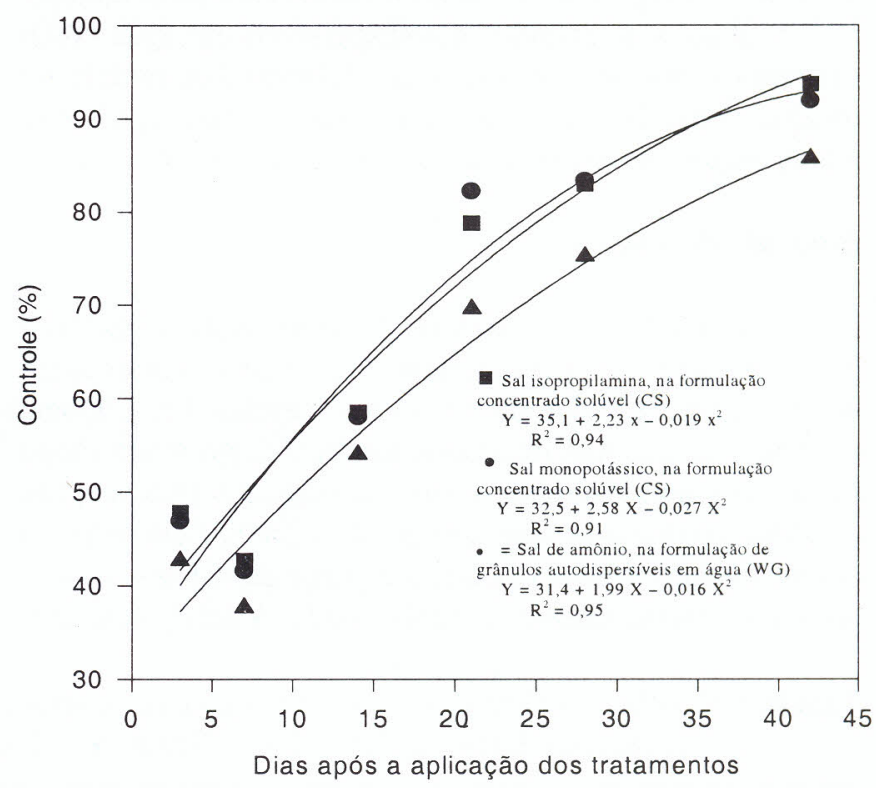

Figura 1. Curva de retenção quadrática e média dos valores originais da percentagem de controle de $\mathrm{Bra}$ chiaria plantaginea, independente do regime de chuva. 
Tabela 2. Controle de Brachiaria plantaginea (\%) por diferentes formulações, sais e doses de glyphosate. Avaliação conjunta dos dados de todas as avaliações. Embrapa Trigo, Passo Fundo, RS. 2001.

\begin{tabular}{lccc}
\hline \multicolumn{3}{c}{ Tratamento } & Controle \\
\cline { 1 - 3 } $\begin{array}{c}\text { Produto } \\
\text { (tipo de sal) }\end{array}$ & Formulação & $\begin{array}{c}\text { Dose } \\
\text { (g /ha e.a.) }\end{array}$ & \\
\hline Monopotássico & CS & 360 & $39,4 \mathrm{~d}^{(1)}$ \\
Monopotássico & $\mathrm{CS}$ & 720 & $46,6 \mathrm{bc}$ \\
Monopotássico & $\mathrm{CS}$ & 1440 & $55,3 \mathrm{a}$ \\
Isopropilamina & $\mathrm{CS}$ & 360 & $38,4 \mathrm{~d}$ \\
Isopropilamina & $\mathrm{CS}$ & 720 & $50,6 \mathrm{ab}$ \\
Isopropilamina & $\mathrm{CS}$ & 1440 & $54,7 \mathrm{a}$ \\
Amônio & WG & 360 & $32,2 \mathrm{e}$ \\
Amônio & WG & 720 & $45,0 \mathrm{c}$ \\
Amônio & WG & 1440 & $50,9 \mathrm{ab}$ \\
Testemunha & - & - & $0,0 \mathrm{f}$ \\
\hline C. V. (\%) & - & - & 9,5 \\
\hline
\end{tabular}

(1) Valores seguidos pela mesma letra, na coluna, não difere entre si pelo teste de Duncan, ao nível de probabilidade de erro de $5 \%$.

\section{Comparação entre o tipo de sal e formulações}

Contrastes de médias de controle da planta daninha conferido pelos produtos em teste revelaram que o sal monopotássico propiciou controle semelhante ao sal isopropilamina, na formulação concentrado solúvel (CS), sendo ambos superiores ao controle obtido com sal de amônio, na formulação de grânulos autodispersíveis em água (WG) (Tabela 3). As características das formulações podem ter resultado em adsorção cuticular e absorção mais rápida pela planta, o que também pode ter reduzido a lavagem pela chuva.

\section{Intervalo de tempo}

A influência do intervalo de tempo entre a aplicação dos tratamentos e a chuva simulada na eficácia da dessecação de B. plantaginea é apresentada nas Tabelas 4 a 7 . Como esperado, os maiores intervalos resultaram em maior efeito tóxico na planta daninha. Assim, na avaliação realizada aos 42 DAT verificou-se que, em geral, o melhor controle foi obtido quando a chuva ocorreu a partir de 4 horas após a aplicação dos tratamentos (Tabelas 4 e 5). A redução de tem- po para menos de 4 horas causou maior redução da eficácia de controle do sal de amônio (formulação WG) comparada ao sal isopropilamina e ao sal monopotássico (ambos na formulação CS). No entanto, o efeito de chuva simulada na eficácia de glyphosate foi reduzido quando a dose do produto foi aumentada, como também foi observado por Bovey \& DiazColon (1969), por Hawf \& Behrens (1974) e por Behrens \& Elakkad (1981), pois maiores quantidades de chuva podem ter sido necessárias para remover maior quantidade de produto da folhagem. Assim, com o aumento da dose de sal de amônio (formulação WG) de $360 \mathrm{~g} /$ ha e.a.para $720 \mathrm{~g} / \mathrm{ha}$ e.a., por exemplo, o controle da planta daninha passou de $29 \%$ para $84 \%$ quando a chuva foi simulada em 1 hora após a aplicação, equivalendo-se estatisticamente ao controle propiciado por sal monopotássico e por sal isopropilamina, ambos na formulação CS (Tabela 4). Doses mais elevadas podem ser responsáveis, também, por absorção mais rápida, de forma que intervalos de tempo mais curtos entre a aplicação e a ocorrência de chuva que os sugeridos, de 6 horas sem chuva após a aplicação, para assegurar a absorção máxima pelas plantas daninhas (Rodrigues \& Almeida, 1998), são suficientes para controle destas.

Quando a chuva foi simulada em 24 horas após a aplicação dos tratamentos, apenas o tratamento com sal de amônio (formulação WG), na dose de $360 \mathrm{~g} /$ ha e.a., ofereceu controle inferior estatisticamente aos demais (Tabela 4).

\section{CONCLUSÕES}

As aplicações de chuvas simuladas antes de 4 horas após a aspersão dos produtos testados reduziram a eficácia no controle da espécie de planta daninha em estudo ( $B$. plantaginea) quando usados na dose de 360 g/ha e.a. O sal de amônio, na formulação de grânulos autodispersíveis em água (WG), sofreu maior redução de eficácia que o sal monopotássico e o sal isopropilamina, ambos na formulação concentrado solúvel (CS).

Independentemente da formulação, o efeito da chuva simulada na eficiência de glyphosate, pode ser reduzido pelo aumento da dose de equivalente ácido.

A formulação determinou a velocidade de controle. $\mathrm{O}$ sal isopropilamina e o sal monopotássico, na formulação concentrado solúvel (CS), apresentaram taxas de controle iguais em termos estatísticos e superiores à do sal de amônio, na formulação de grânulos autodispersíveis em água (WG).

Tabela 3. Contraste estimado para o efeito de sais e formulações de glyphosate, na análise conjunta dos dados de todas as avaliações. Embrapa Trigo, Passo Fundo, RS. 2001.

\begin{tabular}{lccc}
\hline \multicolumn{1}{c}{ Contrastes entre tipo de sal e formulação } & \multicolumn{2}{c}{ Médias } & Probabilidade > F \\
\hline Sal monopotássico CS x sal isopropilamina CS & 47,1 & 47,9 & 0,59 \\
Sal monopotássico CS x sal de amônio WG & 47,1 & 42,7 & 0,03 \\
Sal isopropilamina CS x sal de amônio WG & 47,9 & 42,7 & 0,006 \\
\hline
\end{tabular}


Tabela 4. Efeito do tempo (horas) entre a aplicação dos tratamentos e a irrigação, na eficácia de controle de Brachiaria plantaginea (\%) por diferentes formulações, sais e doses de glyphosate, aos 42 DAT. Embrapa Trigo, Passo Fundo, RS. 2001.

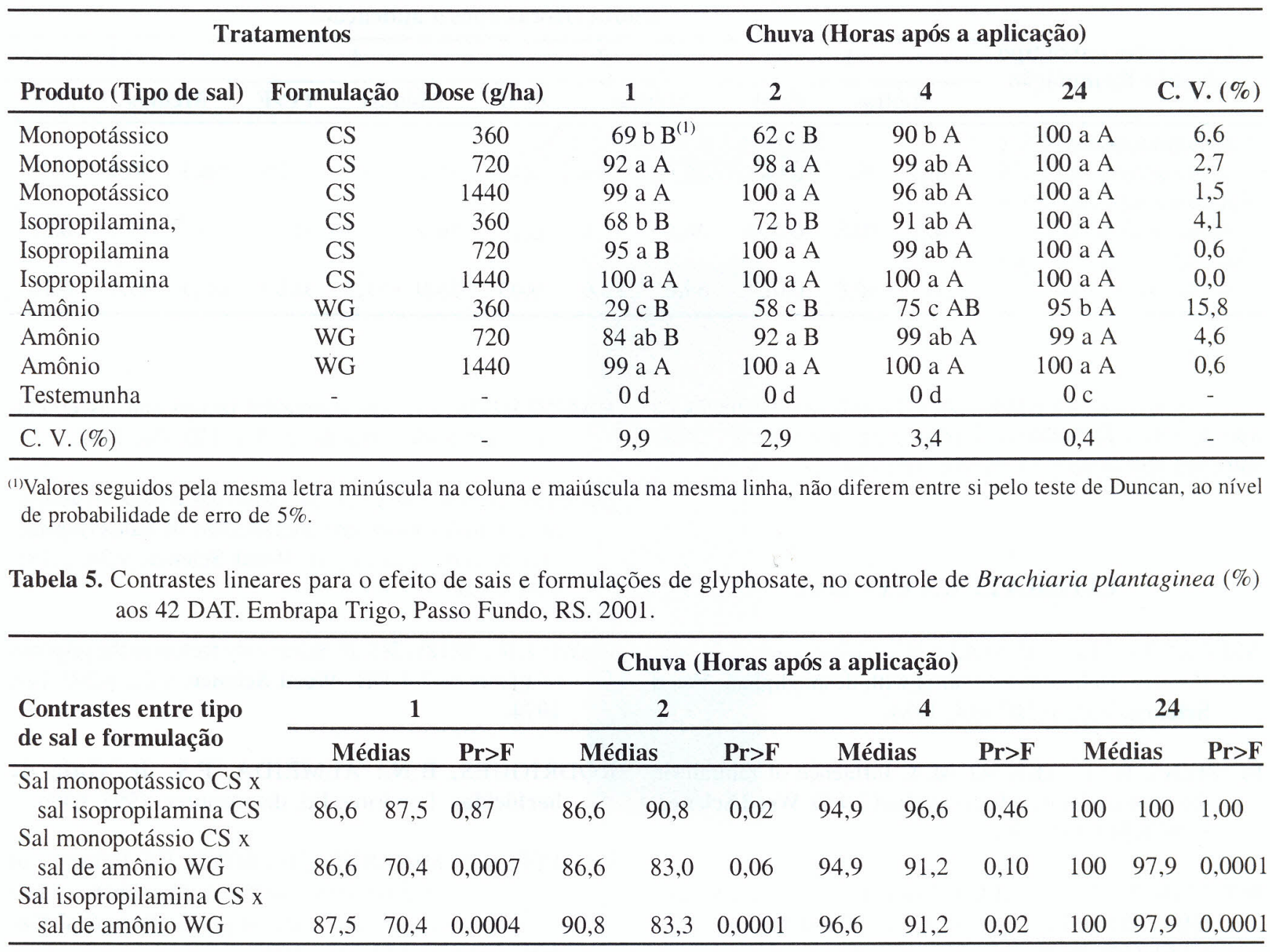

Tabela 6. Efeito do tempo (horas) entre a aplicação dos tratamentos e a irrigação, na eficiência de controle de Brachiaria plantaginea (\%) (análise de dados de todas as avaliações). Embrapa Trigo, Passo Fundo, RS. 2001.

\begin{tabular}{|c|c|c|c|c|c|c|}
\hline \multicolumn{2}{|c|}{ Tratamento } & \multicolumn{4}{|c|}{ Chuva (Horas após a aplicação) } & \multirow[b]{2}{*}{ C. V. $(\%)$} \\
\hline $\begin{array}{c}\text { Produtos } \\
\text { (Tipo de sal e } \\
\text { formulação) }\end{array}$ & $\begin{array}{c}\text { Dose } \\
\text { (g/ha) }\end{array}$ & 1 & 2 & 4 & 24 & \\
\hline Monopotássico CS & 360 & $30,0 \mathrm{~B}^{(1)}$ & $23,7 \mathrm{~B}$ & $50,0 \mathrm{~A}$ & $53,7 \mathrm{~A}$ & 9,2 \\
\hline Monopotássico CS & 720 & $37,5 \mathrm{~B}$ & $36,2 \mathrm{~B}$ & $55,0 \mathrm{~A}$ & $57,5 \mathrm{~A}$ & 10,9 \\
\hline Monopotássico CS & 1440 & $42,5 \mathrm{~B}$ & $50,0 \mathrm{AB}$ & $65,0 \mathrm{~A}$ & $63,7 \mathrm{~A}$ & 8,7 \\
\hline Isopropilamina CS & 360 & $27,5 \mathrm{~B}$ & $23,7 \mathrm{~B}$ & $46,2 \mathrm{~A}$ & $56,2 \mathrm{~A}$ & 7,7 \\
\hline Isopropilamina CS & 720 & $40,0 \mathrm{~B}$ & $42,5 \mathrm{~B}$ & $61,2 \mathrm{~A}$ & $58,7 \mathrm{~A}$ & 10,0 \\
\hline Isopropilamina CS & 1440 & $42,5 \mathrm{C}$ & $52,5 \mathrm{BC}$ & $57,5 \mathrm{AB}$ & $66,2 \mathrm{~A}$ & 7,1 \\
\hline Amônio WG & 360 & $18,7 \mathrm{~B}$ & $20,0 \mathrm{~B}$ & $37,5 \mathrm{~A}$ & $52,5 \mathrm{~A}$ & 12,4 \\
\hline Amônio WG & 720 & $32,5 \mathrm{~B}$ & $31,2 \mathrm{~B}$ & $58,7 \mathrm{~A}$ & $57,5 \mathrm{~A}$ & 8,8 \\
\hline Amônio WG & 1440 & $41,2 \mathrm{~B}$ & $43,7 \mathrm{~B}$ & $57,5 \mathrm{AB}$ & $61,2 \mathrm{~A}$ & 9,6 \\
\hline Testemunha & - & 0,0 & 0,0 & 0,0 & 0,0 & - \\
\hline
\end{tabular}

(1)Valores seguidos pela mesma letra minúscula na coluna e maiúscula na mesma linha, não diferem entre si pelo teste de Duncan, ao nível de probabilidade de erro de $5 \%$. 
Tabela 7. Contrastes lineares para o efeito de sais e formulações de glyphosate no controle de Brachiaria plantaginea (\%) (análise de todas as avaliações). Embrapa Trigo, Passo Fundo, RS. 2001.

\begin{tabular}{|c|c|c|c|c|c|c|c|c|c|c|c|c|}
\hline \multirow{3}{*}{$\begin{array}{l}\text { Contrastes entre tipo } \\
\text { de sal e formulação }\end{array}$} & \multicolumn{12}{|c|}{ Chuva (Horas após a aplicação) } \\
\hline & \multicolumn{3}{|c|}{1} & \multicolumn{3}{|c|}{2} & \multicolumn{3}{|c|}{4} & \multicolumn{3}{|c|}{24} \\
\hline & \multicolumn{2}{|c|}{ Médias } & \multirow{2}{*}{$\begin{array}{c}\operatorname{Pr}>\boldsymbol{F} \\
0,98\end{array}$} & \multicolumn{2}{|c|}{ Médias } & \multirow{2}{*}{$\begin{array}{c}\operatorname{Pr}>\mathbf{F} \\
0,43\end{array}$} & \multicolumn{2}{|c|}{ Médias } & \multirow{2}{*}{$\begin{array}{c}\operatorname{Pr}>\mathbf{F} \\
0,65\end{array}$} & \multicolumn{2}{|c|}{ Médias } & $\operatorname{Pr}>F$ \\
\hline $\begin{array}{l}\text { Sal monopotássico CS x } \\
\text { sal isopropilamina CS }\end{array}$ & 36,7 & 36,7 & & 36,6 & 39,6 & & 56,7 & 55,0 & & 58,3 & 60,4 & 0,38 \\
\hline $\begin{array}{l}\text { Sal monopotássico CS x } \\
\text { sal de amônio WG }\end{array}$ & 36,7 & 30,8 & 0,019 & 36,6 & 31,6 & 0,15 & 56,7 & 51,2 & 0,08 & 58,3 & 57,1 & 0,58 \\
\hline $\begin{array}{l}\text { sal isopropinamina CS X } \\
\text { sal de amônio WG }\end{array}$ & 36,7 & 30,8 & 0,02 & 39,6 & 31,6 & 0,03 & 55,0 & 51,2 & 0,19 & 60,4 & 57,1 & 0,16 \\
\hline
\end{tabular}

A eficácia de glyphosate demonstrou estar ligada ao tipo de sal, à formulação, à dose e ao intervalo de tempo entre sua aplicação e a ocorrência de chuva.

\section{LITERATURA CITADA}

ANDERSON, M.D.; ARNOLD, W.E. Weed control in sunflowers (Helianthus annuus) with desmediphan. Weed Science, v.32, p.310-314, 1984.

BEHRENS, R.; ELAKKAD, M.A. Influence of rainfall on the phytoxicity of foliarly applied 2,4-D. Weed Science, v.29, p.349-355, 1981.

BOVEY, R.W.; DIAZ-COLON, J.D. Effect of simulated rainfall on the herbicide performance. Weed Science, v.17, p.154-157, 1969.
HAMMERTON, J.L. Environmental factors and susceptibility to herbicides. Weeds, v.15, p.330-336, 1967.

HARRISON, S.K.; WAX, L.M. Adjuvant effects on absorption, translocation, and metabolism of haloxyfop-methyl in corn (Zea mays). Weed Science, v.34, p.185195, 1986.

HAWF, L.R.; BEHRENS, R. Selectivity factors in the response of plants to 2,4-DB. Weed Science, v.22, p.245-249, 1974.

RODRIGUES, B.N.; ALMEIDA, F.S. de. Guia de herbicidas. Londrina: Ed. dos Autores, 1998. 648p.

WEAVER, R.J.; MINARIK, C.E.; BOYD, F.T. Influence of rainfall on the effectiveness of 2,4-dichlorophenoxy acetic acid spray for herbicide purposes. Botanical Gazette, v.107, p.540-544, 1970. 